\title{
Adaptive Harmonic Balance Analysis of Oscillators Using Multiple Time Scales
}

\author{
Lei (Lana) Zhu* and Carlos E. Christoffersen ${ }^{\dagger}$ \\ Department of Electrical Engineering, \\ Lakehead University, Thunder Bay, Ontario, Canada P7B 5E1 \\ Email: *1zhu1@1akeheadu.ca and ${ }^{\dagger}$ c.christoffersen@ieee.org
}

\begin{abstract}
A harmonic balance (HB) method to analyze the steady-state response of free-running oscillators is proposed. It does not require a good initial guess of the oscillation frequency and exploits the frequency-domain latency of circuits by using a different number of harmonics in each variable. For the first time the warped multi-time partial differential equation approach is used to simultaneously bring the circuit state to the region of convergence of the $\mathrm{HB}$ analysis and determine the optimum number of harmonics required at each node in the circuit. The analysis of a LC-tuned bipolar oscillator is used to demonstrate the proposed method.
\end{abstract}

Index Terms-Oscillator analysis, Frequency-domain latency, WaMPDE, Adaptive harmonic balance, Multi-time, Steady-state analysis

\section{INTRODUCTION}

The analysis of the steady-state regime of oscillators has been always a hot topic. References [1]-[7] are a few examples. In particular, Harmonic Balance (HB) analysis of oscillators may converge to an unstable equilibrium point or it may not converge at all if a good initial guess of the solution is not provided. Several methods have been proposed to improve this limitation. For example in [6] the HB equations are modified by including the Kurokawa condition to eliminate the DC solution. In [7] a voltage source probe at the fundamental frequency that is an open circuit at all other frequencies is inserted to avoid the DC solution. By means of an iterative process the amplitude and frequency of the probe are adjusted until there is no current through the probe. At this point the autonomous solution is found. A similar probe concept with the addition of a continuation method has been proposed more recently [4] to improve convergence.

Other relevant developments are the exploitation of frequency-domain latency in HB [8], [9] and the use of fast transients to find the steady-state regime [3], [10]. The techniques in [8], [9] take advantage of the fact that in most circuits the number of harmonics necessary to represent each variable (voltage, current) is not the same. By using a different number of harmonics in each variable or compressing the transient behavior, a significant reduction in the computational effort is achieved. In order to accelerate the finding of the steadystate regime, the transient behavior of the circuit is artificially reduced in [10]. In [3], an envelope-transient analysis is used to improve the convergence of the shooting method.
In this paper a robust and fast approach for capturing the steady-state response of oscillators in frequency domain is presented. This approach based on a new adaptive HB technique which is derived from Warped Multi-time Partial Differential Equation (WaMPDE) [2]. This is a technique to separate fast and slow variations in the response of oscillators. This technique provides the following advantages: first, the oscillator frequency is traceable; second, smaller computational effort is required compared with time-consuming transient analysis; and third, a good initial guess of the solution is not necessary. To reduce the computational cost resulting from large amount of harmonics required in some strong nonlinear variables, an adaptive $\mathrm{HB}$ is used to determine the number of harmonics for each state variable separately. Unlike previous implementations, the number of harmonics used in the calculation is always very close to the minimum required for the desired accuracy.

The proposed method is developed in Section II. Section III presents a case study of a LC-tuned bipolar oscillator. Finally, conclusions are summarized in Section IV.

\section{The Proposed Method}

\section{A. Equation formulation}

Let the circuit be represented by its nodal equations:

$$
G u(t)+C \frac{d u(t)}{d t}+\frac{d Q(u(t))}{d t}+I(u(t))=S(t),
$$

here $u(t)$ is the vector of nodal voltages and selected branch currents, $G$ is a matrix of conductances, $C$ is the matrix representing the linear charge terms, $Q(u(t))$ and $I(u(t))$ are vector functions corresponding to the nonlinear devices and $S(t)$ is a vector that represents the sources.

In WaMPDE [2] we must consider at least two time scales: the warped time scale, where the oscillation happens ( $\tau_{1}$ scale) and another time scale equal to the real time ( $\tau_{2}$ scale). In the proposed method, the periodic solution in the warped time $\tau_{1}$ is solved using the HB technique and the solution in the $\tau_{2}$ direction is solved using a time marching technique. This arrangement is usually known as envelope transient analysis. Each element of the $u(t)$ vector is now represented by a function of the two time variables $u_{n}\left(\tau_{1}, \tau_{2}\right)$ ( $n$ denotes 
the node index) and is represented by a set of time-varying phasors,

$$
u_{n}\left(\tau_{1}, \tau_{2}\right)=\Re\left\{\sum_{k=0}^{K} U_{n}^{k}\left(\tau_{2}\right) e^{j k \tau_{1}}\right\},
$$

where $k$ is the harmonic number and the period in the warped time scale $\left(\tau_{1}\right)$ is normalized to $2 \pi$ (i.e., $\omega=1$ ). Equation (1) is now formulated for each frequency:

$$
\left(G+C \Omega_{k}\right) U^{k}+C \frac{\partial U^{k}}{\partial \tau_{2}}+\Omega_{k} Q^{k}+\frac{\partial Q^{k}}{\partial \tau_{2}}+I^{k}-S^{k}=0,
$$

here, $\Omega_{k}$ is a diagonal matrix with nonzero elements equal to $j k \omega_{0}\left(\tau_{2}\right)$, where $\omega_{0}\left(\tau_{2}\right)$ is the unknown fundamental frequency. The $Q^{k}, I^{k}$ and $S^{k}$ vectors are all functions of $\tau_{2}$. The first two are normally evaluated using the discrete Fourier transform (DFT). Equation (2) is discretized in the $\tau_{2}$ direction using the backward Euler (BE) rule. The resulting algebraic nonlinear system is then solved with the Newton-Raphson method for each value of $\tau_{2}$.

The fundamental frequency $\omega_{0}\left(\tau_{2}\right)$ is an additional unknown that must be calculated for each value of $\tau_{2}$. The phase of one of the variables must then be fixed to restore the number of unknowns to be equal to the number of equations. That is achieved by setting the imaginary part of one of the variables to be zero,

$$
\Im\left(U_{n}^{1}\right)=0 .
$$

\section{B. Adaptive HB Oscillator Analysis}

The simulation is started with an initial condition equal to the DC bias point. An excitation current,

$$
i_{S}\left(\tau_{1}, \tau 2\right)=\left\{\begin{array}{ll}
I_{0} \cos \left(\tau_{1}\right) & \text { if } \tau_{2} \leq t_{a} \\
0 & \text { otherwise }
\end{array},\right.
$$

is injected from the ground node into one of the nodes where oscillations are expected. Here, $I_{0}$ is a small real number (normally a few $\mu \mathrm{A}$ ) and $t_{a}$ is set equal to the initial timestep size along $\tau_{2}(h)$. The purpose of $i_{S}$ is to start oscillations by moving the system away from the equilibrium point. The system will then naturally tend to reach the desired oscillatory steady-state. The key of the work presented here is first to reach a point in $\tau_{2}$ close to the steady state in the minimum possible number of Newton iterations and then use the state at that point as the initial guess of a regular autonomous $\mathrm{HB}$ analysis. Since the focus is on the steady-state it is not necessary to calculate the transient evolution with great precision as long as it converges close to the actual steadystate of the circuit.

In order to minimize the number of iterations required to get close to the steady-state the following considerations are taken. First, the BE rule is used. It is known that this integration method introduces numerical damping. The effect of this is that any oscillations along the $\tau_{2}$ (not along the $\tau_{1}$ scale) scale are damped [11] and this is beneficial since it allows the use of a longer time step. Second, the tolerance of the Newton method is adaptively controlled during the transient evolution to prevent it from being unnecessarily small. This has the effect of reducing the number of Newton iterations that are necessary at each value of $\tau_{2}$. The Newton tolerance (tol) is set to

$$
\text { tol }=\max \left\{0.1 \% U_{\max }^{k}, 10^{-7}\right\},
$$

where $U_{\max }^{k}$ is the amplitude of the largest oscillation in the circuit at the previous value of $\tau_{2}$. Third, an adaptive time step algorithm is employed. The algorithm chooses the size of $h$ based on the number of Newton iterations required to achieve the desired tolerance. No truncation error checking is necessary in this case and so the number of necessary time steps is reduced.

It is assumed that the solution is very close to the steady state when the local frequency function has become constant and the difference between two periodic solutions along $\tau_{2}$ becomes small,

$$
\left\|\frac{U_{n}\left(\tau_{2}+h\right)-U_{n}\left(\tau_{2}\right)}{U_{n}\left(\tau_{2}+h\right)}\right\|<\epsilon_{1},
$$

where $\epsilon_{1}$ is a small relative tolerance. If $U_{n}\left(\tau_{2}+h\right)$ is very close to zero an absolute tolerance $\epsilon_{2}$ is used. At this point the envelope transient is stopped and a regular oscillator HB analysis is started using the last solution along $\tau_{2}$ as the initial guess. A small tolerance is used this time to achieve the desired accuracy (typically on the order of $10^{-9}$ ). Note that the regular oscillator $\mathrm{HB}$ analysis is obtained by setting all derivatives respect to $\tau_{2}$ to be zero in (2). There is no need for probes or any other special provisions since the initial guess is very close to the steady-state solution. Normally only a few additional Newton iterations are needed.

Another provision to improve the efficiency of the simulation is to adaptively control the number of harmonics for each variable $u_{n}$ independently. At $\tau_{2}=0$, only a few harmonics have to be considered because the oscillations are started with DC bias points. In our proposed method, only three harmonics are considered initially. At the end of the calculation for each value of $\tau_{2}$, the values of the last two harmonics are considered. If they are greater than some threshold value then the number of harmonics for that variable is increased by one, otherwise it is left unchanged for the next time step. One advantage of this approach is that the number of harmonics is increased as needed, i.e. there is no need to perform an initial calculation with many harmonics to later decide the ones that must be removed. It is then important to start with a small oscillation value at $\tau_{2}=0$. Each row of (2) (nodal equation at one frequency) is considered at a number of frequencies equal to the number of harmonics of the corresponding nodal voltage. In this way the number of equations is always kept equal to the number of unknowns.

\section{CAse Study And Discussion}

The steady-state analysis of a LC-tuned oscillator employing the proposed method is presented in this section. Fig. 1 shows 


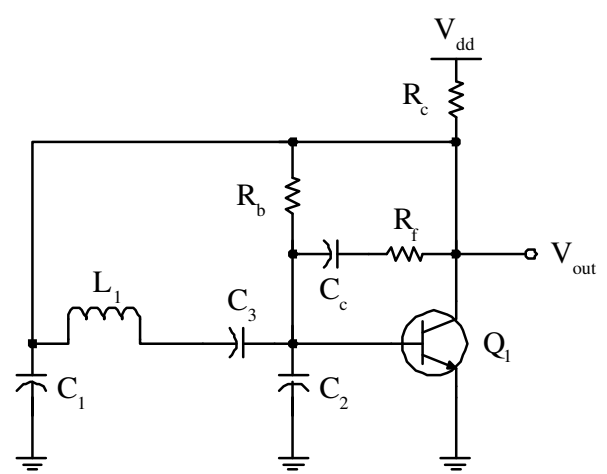

Fig. 1. LC-tuned bipolar oscillator.

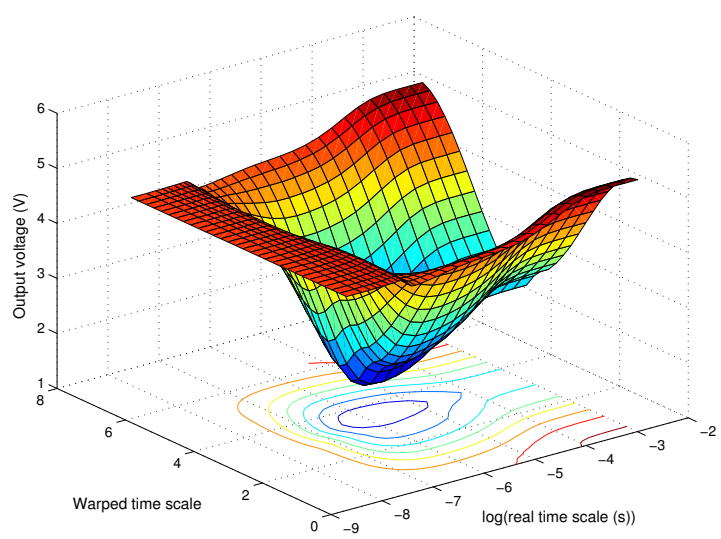

Fig. 2. Bi-dimensional representation of output voltage.

the schematic of a LC-tuned bipolar oscillator, which was one of the autonomous circuit examples in [3]. In this circuit $C_{1}=$ $C_{2}=33 \mathrm{pF}, C_{3}=3.17 \mathrm{pF}, C_{c}=560 \mathrm{pF}, L_{1}=100 \mathrm{nH}$, $R_{f}=680 \Omega, R_{b}=100 \mathrm{k} \Omega, R_{c}=1.2 \mathrm{k} \Omega$ and $V_{d d}=10 \mathrm{~V}$.

The excitation current $\left(i_{S}\right)$ was injected to the base node and $I_{0}$ was set to $10 \mu \mathrm{A}$. This oscillator exhibits a very slow initial transient compared with the oscillation period but only a few time steps along $\tau_{2}$ are necessary since the oscillations and the transient variations are decoupled in two different time scales. The stop time along $\tau_{2}$ is $1.0326 \times 10^{-3}$ seconds. The bi-dimensional plot of the output voltage as a function of $\tau_{1}$ and $\tau_{2}$ is shown in Fig. 2. A logarithm scale was used along $\tau_{2}$ to improve the visibility of the samples along that axis. Note that the initial time step is chosen very short but the adaptive algorithm quickly increases its size and thus a only few points are necessary to cover the entire simulation interval.

The adaptive control of the time step along $\tau_{2}$ keeps the number of Newton iterations small for each value of $\tau_{2}$ (typically $2-4$, see Fig. 5). The oscillator frequency determined by the warped function $\omega_{0}\left(\tau_{2}\right)$ as shown in Fig. 3 is $308.87 \mathrm{MHz}$. Fig. 3 demonstrates the robustness of the method. Different initial frequencies far away from the solution quickly converge

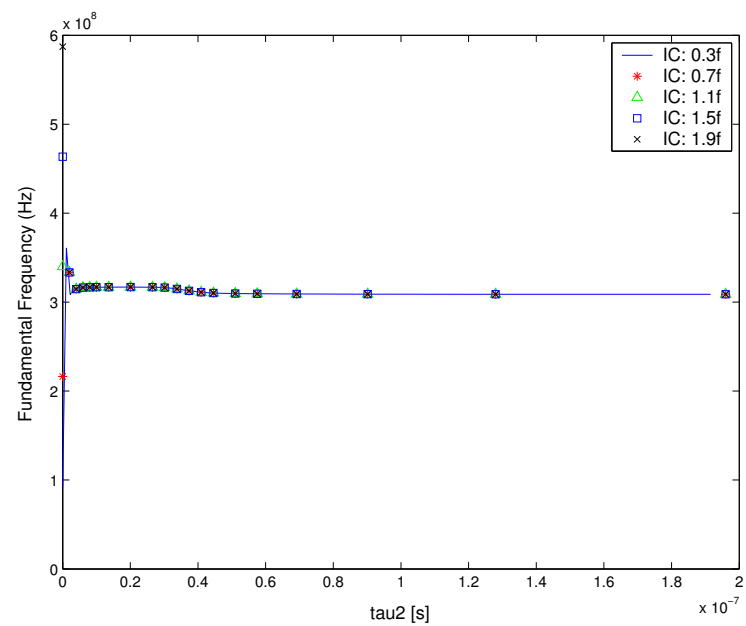

Fig. 3. Fundamental frequency as a function of the real time scale $\left(\tau_{2}\right)$.

to the correct value and become constant in the asymptotic steady-state stage.

The tolerance of the HB analysis following the multitime simulation was set to $10^{-10}$. Only 3 Newton additional iterations are necessary to obtain the steady-state solution with the initial guess provided by the multi-time simulation. In Fig. 4 the steady state is shown together with the final multitime result. The match is so close that it is hard to tell them apart.

Fig. 5 shows the Jacobian matrix size and the number of Newton iterations at each time step (line number). It can be observed that few iterations are necessary at each time step. As mentioned before this is due to the adaptive time step control algorithm. The Jacobian matrix size increases as the number of harmonic increase. If a fixed number of harmonics is considered the size of the Jacobian matrix is $116 \times 116$. It is obvious that the new approach drastically reduces the Jacobian matrix for small values of $\tau_{2}$ and saves considerable computational effort. The use of variable number of harmonics in this case increases the computational speed by a factor of ten.

If $R_{f}$ in the circuit of Fig. 1 is reduced to $200 \Omega$, the oscillation period eventually doubles and this originates a subharmonic component as shown in Fig. 6. The proposed method is not able to detect this condition. The detection of subharmonic generation and chaos in a WaMPDE simulation is a topic for future research.

\section{CONClusions}

We have presented a new approach to determine the steadystate of free-running oscillators that is robust and efficient. This new method does not require a good initial guess of the oscillation frequency and incorporates several ideas to improve robustness and reduce the computational cost. The method uses the WaMPDE approach to naturally bring the circuit state to 


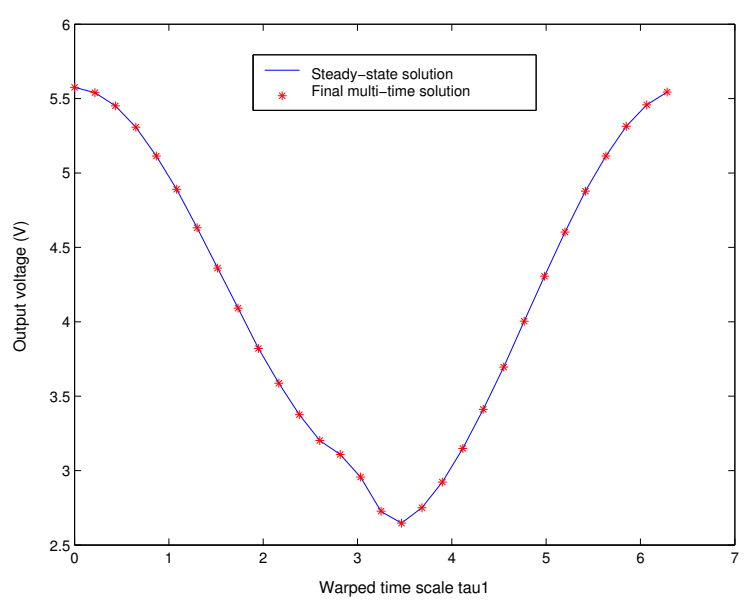

Fig. 4. Steady-state solution compared to final multi-time solution.

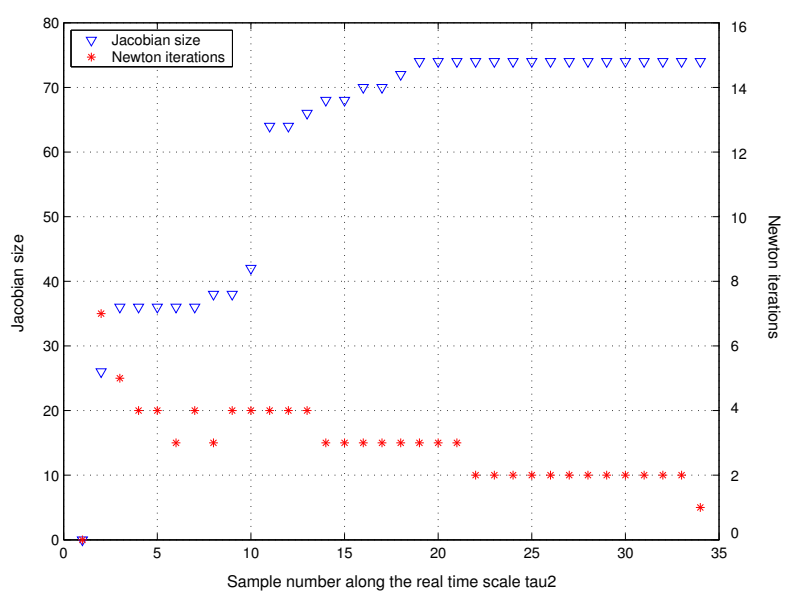

Fig. 5. Jacobian size and number of Newton iterations.

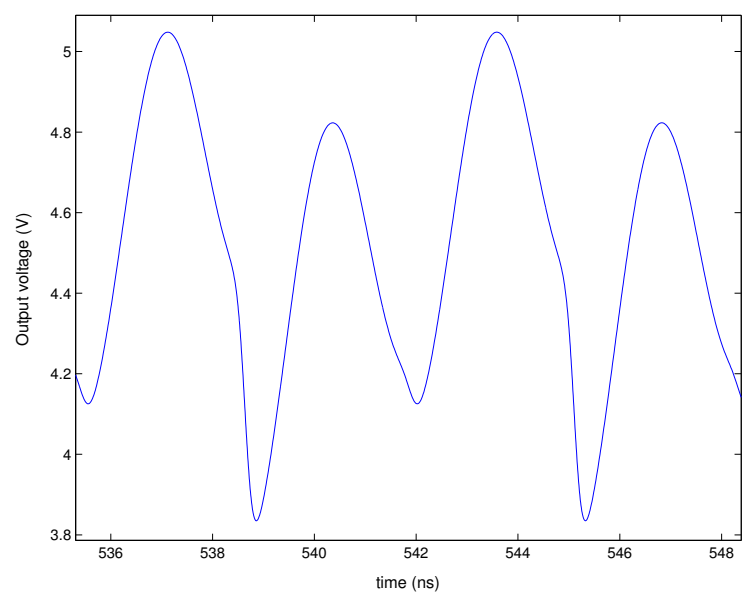

Fig. 6. Transient simulation with $R_{f}=200 \Omega$ showing subharmonic generation. the region of convergence of the HB analysis. It was shown for the first time that the transient evolution along the slow time axis provide the optimum conditions to determine the minimum number of harmonics required at each node in the circuit. As a result of this adaptive scheme the Jacobian matrix in the Newton method is reduced and the simulation is faster. Both the oscillation frequency and the steady-state solution of each state variable are obtained in a fast and accurate way.

\section{ACKNOWLEDGMENT}

The authors would like to acknowledge the support of Lakehead University and Science and Engineering Research Canada (NSERC).

\section{REFERENCES}

[1] A. Collado, F. Ramírez, A. Suarez and J. P. Pascual, "Harmonic balance analysis and synthesis of coupled-oscillator arrays," IEEE Microwave and Wireless Components Letters, vol. 14, no. 5, March 2004, pp. 192194.

[2] O. Narayan and J. Roychowdhury, "Analyzing oscillators using multitime PDEs,"IEEE Transactions on Circuits and Systems-1: Fundamental Theory and Applications, vol.50, no.7, Jul. 2003, pp. 894-903.

[3] A. Brambilla and P. Maffezzoni, "Envelope-following method to compute steady-state solutions of electrical circuits,"IEEE Transactions on Circuits and Systems-1: Fundamental Theory and Applications, vol.47, Jul. 2000, pp. 999-1008.

[4] M. Gourary, S. Ulyanov, M. Zharov, and S. Rusakov, "Simulation of high-Q oscillators," 1998 IEEE/ACM ICCAD Digest, 1998, pp. 162-169.

[5] V. Rizzoli, A. Constanzo and A. Neri, "Harmonic balance analysis of microwave oscillators with automatic suppression of degenerate solution," Electronic Letters, vol. 28, no. 3, Jan. 1992, pp. 256-257.

[6] C. Chang, M. B. Steer, S. Martin and E. Reese, "Computer-aided analysis of free-running microwave oscillators," IEEE Trans. on Microwave Theory and Techniques, vol. 39, no. 10, Oct. 1991, pp. 1735-1745.

[7] E. Ngoya, A. Suarez, R. Sommet and R. Quéré, "Steady state analysis of free or forced oscillators by harmonic balance and stability investigation of periodic and quasi-periodic regimes," Int. J. of Microwave and Millimiter-Wace CAE, vol. 5, no. 3, 1995, pp. 210-223.

[8] H. Asai, H. Makino, "Frequency domain latency and relaxation-based harmonic analysis of nonlinear circuits,"Proc. of the 34th Midwest Symp. on Circuits and Systems, vol. 1, May 1991, pp. 202-205.

[9] M. Gourary, S. G. Rusakov, S. L. Ulyanov, M. M. Zharov, K. K. Gullapalli, B. J. Mulvaney, "A new technique to exploit frequency domain latency in harmonic balance simulators,"Proceedings of the 1999 Design Automation Conference, vol. 1, Jan. 1999, pp. 65-68.

[10] R. Larchevéque and E. Ngoya, "Compressed transient speeds up the periodic steady state analysis of nonlinear microwave circuits," 1996 IEEE MTT-S Digest, pp. 1369-1372.

[11] J. Roychowdhury, "Making Fourier-envelope simulation robust," 2002 IEEE/ACM ICCAD, pp. 240-245. 